\title{
Design of pedestrian network friendliness maps
}

\author{
Nuno Sousa, $\mathrm{PhD}, \mathrm{MSc}$ \\ INESC-Coimbra, Coimbra, Portugal \\ Department of Sciences and Technology, Open University, Lisbon, Portugal \\ R. da Escola Politécnica 141-147, 1269-001 Lisboa, Portugal. Phone +351 213916300 \\ nsousa@uab.pt \\ ORCID 0000-0002-2681-5035 \\ Filipe Pais, PhD student, MSc, CEng \\ INESC-Coimbra, Coimbra, Portugal \\ Department of Civil Engineering, Faculty of Sciences and Technology, University of Coimbra, \\ Coimbra, Portugal \\ Rua Luís Reis Santos - Polo II, 3030-788 Coimbra, Portugal. Phone +351 239797100 \\ filipejmcpais@gmail.com \\ ORCID 0000-0002-9802-0338 \\ Eduardo Natividade-Jesus, PhD, MSc, CEng \\ Department of Civil Engineering, Polytechnic Institute of Coimbra, Portugal. \\ INESC-Coimbra, Coimbra, Portugal \\ ednativi@isec.pt \\ ORCID 0000-0001-6213-386X \\ João Coutinho-Rodrigues, $\mathrm{PhD}, \mathrm{MSc}$, CEng \\ INESC-Coimbra, Coimbra, Portugal \\ Department of Civil Engineering, Faculty of Sciences and Technology, University of Coimbra, \\ Coimbra, Portugal \\ Rua Luís Reis Santos - Polo II, 3030-788 Coimbra, Portugal. Phone +351 239797100 \\ coutinho@dec.uc.pt \\ ORCID 0000-0001-9311-5584
}

\begin{abstract}
:
This article introduces the concept of pedestrian, or walking friendliness, and presents a methodology for obtaining maps thereof. Walking friendliness is a quality of walking indicator, defined for any given origin in a city, which combines accessibility measures, based on impedance between that origin and destinations, with performance scores for the pedestrian infrastructure linking those origins and destinations. The methodology uses geographic information systems to obtain walking friendliness values and represent them in a map. The approach is demonstrated through a case study for the city of Coimbra, Portugal, for which friendliness maps were derived. The procedure and maps that were produced can be scaled to any size of city.
\end{abstract}

Keywords: infrastructure planning, municipal \& public service engineering, town \& city planning 


\section{Introduction}

The last decades have witnessed an increasing interest of researchers, municipal authorities and the general public in active travel modes, like walking or cycling, prompted by sustainability worries, traffic congestion and health issues (Hope-Dunbar and Huxford, 2005; Woodcock et al., 2007; Donovan, 2015; Tight, 2016; Rassafi and Mohajeri, 2017). Walking, the most basic transport mode, increases physical activity, is non-polluting and socially inclusive. Walking always has a considerable share among all transport modes, for people of all ages and social status, especially for small distances. As such, it is important to evaluate how well a city's transport infrastructure is prepared to support this mode.

Pedestrian infrastructure provides support for the walking mode. Performance of this infrastructure can be assessed for the quality of service provided to pedestrians using so-called performance indicators. These indicators usually evaluate infrastructure performance on a per-network arc basis and take into account multiple aspects, such as structural condition, walking comfort, safety from traffic, etc., often combining them into one value (i.e. each network arc is given a performance score). Since arc-based performance indicators are disconnected from the actual pedestrian trips, and because not all trips are of the same importance, frequency or pedestrian demand, constructing more wide-ranging quality of walking indicators that go beyond engineering aspects requires combining performance and trips, which in turn requires including accessibility concepts in the analysis.

In this context, the present article introduces the concept of pedestrian or walking friendliness (WF) index, as a quantitative quality of walking indicator, based both on engineering aspects of the network arcs and their role in serving people going from origins to destinations. It combines the aforementioned per-arc based performance indicators with the notion of accessibility as a cost (lower values are better) (Geurs and Rietsma van Eck, 2001; Reggiani et al., 2011) and is locally defined for each point of the urban space under consideration. In this article, accessibility as cost is understood, for origin $i$, as

$$
A_{i}=\sum_{j} I^{(1)}{ }_{i j}
$$

with $I^{(1)}{ }_{i j}$ the impedance, i.e. a measure of the difficulty of going from that origin $i$ to the closest destination of type $j$. Formula (1) inspired the WF index concept, even though it was not used explicitly in the calculations.

The methodology for obtaining a WF index requires several datasets and makes use of geographical information systems (GIS) to carry out the calculations and represent results. The outcome of the methodology is a map (or set of maps) in which, for each origin, the WF index is displayed in colourcode. These maps provide a global view of the pedestrian network quality, which combines individual arc performance with accessibility and pedestrian demand considerations. The methodology is thus a novel way to assess pedestrian network quality and its outcome can be used by municipal decision makers as a basis to plan for interventions aiming at improving the infrastructure.

\subsection{Literature review}

Performance indicators for the pedestrian infrastructure include e.g. Gallin (2001), TRL (2003), Landis et al. (2001), and Khisty (1994). More recent methods were put forward by Sousa et al. (2017) and Corazza et al. (2016). See also Aghaabbasi et al. (2017) and references therein for other recent indicator proposals and Aghaabbasi et al. (2018) for an evaluation of the capability of walkability audit tools to assess 
sidewalks. Performance indicators use different methodological approaches to yield a per-arc performance value for the pedestrian infrastructure considering different attributes such as e.g. sidewalk condition, safety, walking environment, etc.

Concerning the subject of pedestrian accessibility, van Wee (2016) notes that traditionally the accessibility literature has not focused much on short distances and slow modes. However that author also recognises that the interest in this field rose significantly in the last decade, as shown by the work carried out by Forsyth and Krizek (2010), who analysed the results of over 300 studies to determine the most effective strategies to foster the use of active modes; Manaugh and El-Geneidy (2013), who studied the relationship among satisfaction levels, motivations, and home location factors of walkers; and Moniruzzaman et al. (2014), who proposed the 'Compliance Potential Map', a GIS map overlay tool which combines trip distance and frequency to assess the potential contributions of walking towards physical activity. Other, more recent examples include the study of Liang et al. (2017) on walking accessibility to urban parks; Yang et. al (2018), who explored the relationship between pedestrian accessibility and property prices; and Saghapour et al. (2018), who used regression models to show that incorporating accessibility measures in active transport modelling improves demand forecasting.

In the examples above, the subjects of performance indicators and pedestrian accessibility were treated separately. Research combining them is scarcer. Recent examples include the study of Koh and Wong (2015) on preferences of active travel mode users in last mile trips to public transport stations, together with condition and safety of the supporting infrastructure. Walkability is an important concept, which considers the multiple aspects influencing walking, and quality of walking, in a given area (e.g. Kelly et al., 2011; Manaugh and El-Geneidy, 2011; Ewing and Handy, 2009). Walkability involves many factors, including land-use, street design, walking environment, and health-related issues, along with more technical aspects such as sidewalk performance or adequacy of intersection facilities for pedestrians, and is often measured in a quantitative way (see e.g. Lwin and Murayama, 2011). In this sense, the WF index can be seen as a quantitative walkability indicator, at full city scale, which focuses on the pedestrian network and how well it provides access to important destinations.

\section{Methodology}

The main idea of the WF index is to define and evaluate a quantity which represents the average pedestrian network performance a person experiences in her walking trips from origins to destinations, weighted by trip importance.

The methodology considers fixed origins and attractor points of the city, i.e. urban facilities, segregated by type, as destinations. The walking trips considered are those going from each origin $i(i=1, \ldots, M)$ to the closest facility of each type $j(j=1, \ldots, N)$, and the WF index is thus calculated for each origin, i.e. it is $W F_{i}$. Note that under the methodology job locations are not considered as an attractor and do not feature in the destinations list. This is because pedestrians have a fixed job; they do not have the option to walk to their "closest job", as they do when selecting e.g. a restaurant or grocery store. Furthermore, while in general shorter commuting times lead to higher job satisfaction (Chatterjee et al., 2017), other researchers found that some people do not actually want a very short commuting distance to their job (Ory et al., 2004); having commerce and service activities such as e.g. schools, kindergarten, grocery shops, and 
basic medical services available close to home is likely to be more important (van Wee, 2016; Boarnet et al., 2011). Because there are many sorts of walking trips, and motivation and demand therefor, not all trips have the same importance. For this reason an importance weighting factor $\omega_{j}$ is defined for each facility type $j$. It is possible to apply the methodology with origin-dependent weighting factors (i.e. $\omega_{j} \rightarrow$ $\left.\omega_{i j}\right)$ if the pedestrian demand is known in detail for each origin. This would yield more precise results and also make it possible to include job locations. In practice however, this data is seldom available.

Travelling between origins and destinations is done using the pedestrian network. Usually this network shares space with the streets network to a very high percentage, but may occasionally have arcs of its own (see Melia and Shergold (2018) for a discussion on the politics and implications of road restrictions). The methodology requires the pedestrian network to be endowed with information concerning its performance, which needs to be collected from field surveys on a per-arc basis. Because all arcs in the study area need to be evaluated and streets networks typically have many arcs, it is necessary to use a simple and practical performance evaluation method. Performance is affected to a significant degree by the way a network arc shares space with other transport modes, such as cycles or motor vehicles, as poorly designed shared space can be unwelcoming, uncomfortable, or even frightening (Kang and Fricker, 2016). As such, the importance of carefully planning these spaces to cater for all transport modes cannot be understated. In particular, given speed differences between walking and other modes, route segregation is often necessary (Banister and Hickman, 2013; Parkin, 2018). See Moody and Melia (2015) for a breakdown on shared space issues, including pedestrian aspects.

Finally, to carry out the calculations that lead to the WF index, a GIS environment is necessary, along with the datasets with relevant information.

\subsection{Datasets required}

The methodology requires having three datasets as feature classes in the GIS environment, namely a set of origins, a set of destinations, and a pedestrian network. Feature classes are basic GIS objects, collections of standard features (points, polylines, polygons, etc.) representing items in the field (e.g. locations, streets, areas, etc.), whose attributes are stored in associated tables. A terrain map should also be added, for better visualisation of the results.

The recommended set of origins is the set of centroids (geometric centres) of a quadrangular grid, or mesh created over the city in the GIS environment using geoprocessing tools. This is more general than using as origins e.g. residential buildings, as it covers the whole of a city.

The set of destinations is, as mentioned, the set of urban facilities considered for the case at hand. Table 1 below shows a possible list of facilities which can be considered and proposed weighting factors. Higher weights mean trips to the corresponding facilities are likely to be more frequent. 


\begin{tabular}{|c|c|c|}
\hline $\begin{array}{c}\text { Higher importance facilities } \\
\omega_{j}=3\end{array}$ & $\begin{array}{c}\text { Medium importance facilities } \\
\omega_{j}=2\end{array}$ & $\begin{array}{c}\text { Lower importance facilities } \\
\omega_{j}=1\end{array}$ \\
\hline Primary schools, kindergartens (UK: up to KS2) & College (UK: KS5) & Post office \\
\hline Secondary schools (UK: KS3-4) & Shopping centres & Police office \\
\hline Grocery stores & Entertainment Lounges & Sports centres \\
\hline Bakeries & Primary healthcare centres & Cultural and sports associations \\
\hline & Pharmacies & Higher education facilities \\
\hline & Restaurants & Elderly care centres \\
\hline & & Churches \\
\hline & & City Hall \\
\hline & & Parish \\
\hline
\end{tabular}

Table 1. Facility importance weights

This list is not exhaustive; different types of facilities could be considered, as well as other weighting factors. The latter usually depend on cultural and regional aspects, so they should be chosen on a case-bycase basis.

With respect to the pedestrian network, information on impedance values $(I)$ and performance scores $(P)$ is necessary for each arc. Commonly used impedances are distance or time, but other types can be considered (e.g. walking energy expenditure). For an arc, its impedance is defined as the difficulty in going through it. If the distance is used as impedance, the network arcs, which have two directions, usually named "From-To" and "To-From", will have the same impedance values. If other types of impedances are used, those two values may differ. As for performance, it should be noted that this quantity can be measured in any sensible way but, to be used in the proposed methodology, that information needs to be translated into a quantitative scale.

\subsection{Methodology implementation}

The practical GIS implementation of the procedure is best described in steps, as follows. In the steps below, the terminology of the ESRI ArcGIS environment is used. Other GIS environments may use different terms, but the correspondence should be clear from the descriptions given.

1. Create/obtain the GIS feature classes of the required datasets. The origins feature class can be created using GIS geoprocessing tools to generate a square mesh of a given size (e.g. $50 \mathrm{~m} \times 50 \mathrm{~m}$ ) over the city map (polygon feature class), together with its associated centroids. The centroids form the origins feature class.

2. For the origins feature class, use GIS geoprocessing tools to evaluate the straight-line distance from each origin to the closest arc of the pedestrian network (snapping distance) and store this distance in the origins feature class associated table. Manually delete from the origins feature class all the centroids (and associated polygons) with distances greater than a certain value, larger than the mesh size. This is necessary to avoid imprecisions due to excessive origin-to-network snapping distance, which is not considered in GIS network analysis. It also has the effect of visually clearing up the map from zones without nearby pedestrian infrastructure. These are usually zones without buildings or green space parcels of land.

3. For the pedestrian network feature class, add columns to its associated table containing, for each arc, 
the product $(K)$ of impedance $\times$ performance value in the From-To and To-From directions, i.e. $K_{F T}=$ $I_{F T} \times P$ and $K_{T F}=I_{T F} \times P$. These $K$ values can be obtained using GIS scripting tools (ArcGIS: field calculator tool, which uses Python or VBA as scripting languages). If the network is undirected, that is to say, if the From-To and To-From directions lead to the same impedance, one such value suffices.

4. For each origin $i$ and each destination type $j$, use GIS network analysis tools (ArcGIS: closest facility) to find the shortest-impedance path from the origin to the closest facility of type $j$ and set $K$ as accumulation variable in the calculations (an accumulation variable is a quantity in the network feature class associated table, whose value is stored cumulatively, in tandem with impedance, as the shortest-impedance path is derived). Use a facility search tolerance distance similar to the one used for removing origins in step 2 (the facility search tolerance is the maximum allowed snapping distance between that facility and the pedestrian network). Repeat this procedure for each destination type $j$ and store the cumulative value $K_{i j}$ in the associated table of the origins feature class, as well as the shortest-impedance path cumulative impedance value $\left(S_{i j}\right)$. The $K_{i j}$ mean 'cumulative performancepondered impedance from origin $i$ to the closest facility of type $j$ '. In the end, the origins feature class associated table will have $2 N$ extra columns, one containing $K_{i j}$ and the other $S_{i j}$.

5. For each destination type, add one column to the origins feature class associated table (total of $N$ extra columns) and use GIS scripting tools to fill it with the quotient $\bar{K}_{i j}=K_{i j} / S_{i j}$. This quotient is the impedance-averaged pedestrian network performance in going from $i$ to $j$.

6. In the origins feature class add one column and use GIS scripting tools to fill it with $W F_{i}=\Sigma_{j} \omega_{j} \bar{K}_{i j} / \Sigma_{j} \omega_{j}$. This quantity is the WF index for centroid $i$ and is the final outcome of the calculations. It has the interpretation of impedance-averaged pedestrian network performance in going from origin $i$ to the closest facilities of all types, weighted by facility importance'. Note that the WF index is not a performance indicator per se; rather, it reflects average performance scores, in the same scale.

7. Use GIS join tools to associate the $W F_{i}$ values to the corresponding $i$ in the mesh polygon feature class. Represent the polygon feature class joined values in the city map in colour-code or greyscale.

\section{Case study and results}

The methodology was applied to a practical case study, the city of Coimbra, Portugal. This is a mid-sized city in the centre of Portugal, with circa 130000 inhabitants (census 2011) and health and higher education as main economic activities. The methodological procedure was carried out using the ESRI ArcGIS GIS environment.

The origins feature class was based on a $50 \mathrm{~m} \times 50 \mathrm{~m}$ mesh size and centroids more than $150 \mathrm{~m}$ away from the pedestrian network were removed. The destinations feature class was available from previous projects, as well as the pedestrian network. Figure 1 shows a map representing the three feature classes. 


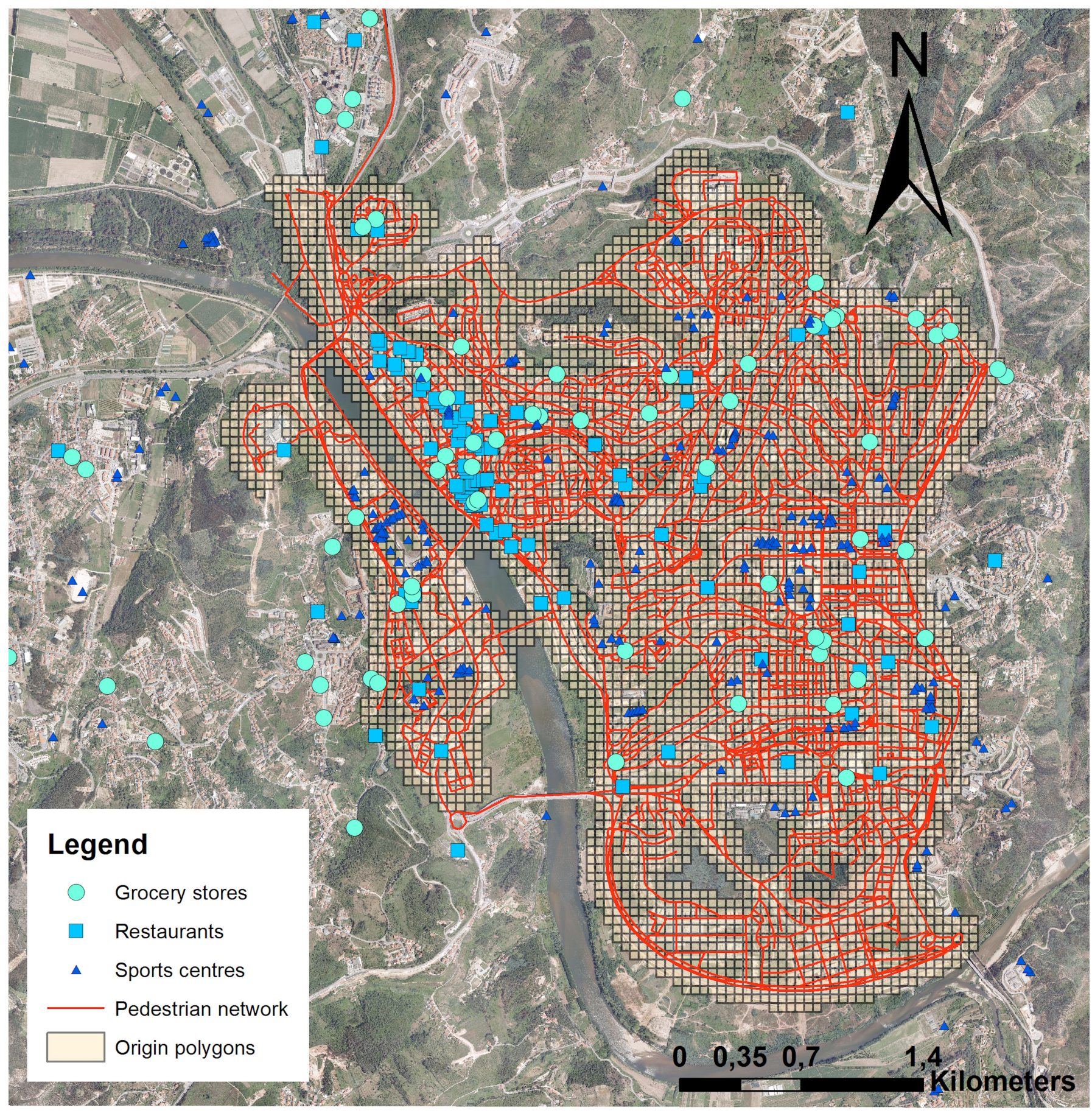

Figure 1. Case study GIS feature classes: origins, destinations (three types) and pedestrian network

Centroids of the square mesh form the origins feature class and the remaining lines are the pedestrian network. Dots indicate locations of three types of urban facilities (destinations). To avoid clutter, no more facility types were displayed. 




Figure 2. Pedestrian network performance.

A city-scale survey was conducted following the sidewalk multicriteria assessment method of Sousa et al. (2017) to obtain pedestrian network performance data, in four ordinal performance classes. As required by the WF methodology, these classes were made quantitative using a discrete 1-4 Likert scale, with $4=$ best. The 'safety' criteria weights mentioned in that reference were used, with a few minor adjustments. 
These were chosen over other possible weights values because they put emphasis on streets network space-sharing issues. A total of 3730 arcs were surveyed, covering over $250 \mathrm{~km}$ of sidewalks. The associated time cost was of approximately four persons-month, showing scalability of the assessment methodology to full city size. Figure 2 shows a GIS-generated map of the performance of those individual arcs. A facility search tolerance of $150 \mathrm{~m}$ was used.

This performance data was added to the pedestrian network feature class and the facilities types and weights of Table 1 were considered. Two impedances types, distance and penalised distance, were considered, both leading to undirected networks. The results are now shown.

\subsection{Results: distance as impedance}

The first impedance type considered was the (arguably) most common impedance measure, distance (network arc length). Results for this impedance were derived following section 2.2 and the WF index output is presented in Figure 3 below. Note that, since performance takes values in the [1,4] interval, so does the WF index, by definition.

Looking at the figure, and comparing it with Figure 1, it can be seen that places with lower WF score lie around long streets with poor pedestrian performance score. Many of these poor scores are due to high traffic volume and unsuitable separation between motor vehicles and pedestrians.

Because walking trips consider the closest facility as destination, it is natural that WF scores correlate with network performance scores of nearby streets. However, the WF map shows that all trips considered, the combined effect of having nearby poor/good performance streets nearby is not easily predictable just by looking at the network arc performance values. WF index calculations need to be explicitly carried out to have a clear idea of how a particular origin (or zone) fares in pedestrian friendliness. The WF index is, therefore, a more wide-reaching, aggregate indicator and this is its main plus-value, a plus-value which makes it preferable to mere network performance indicators as a quality of walking evaluation tool.

It is important to stress that the WF index is not an accessibility index; it does not measure how close destinations are. Rather, it merely uses impedance to destinations to determine what paths pedestrians use, and then evaluates infrastructure performance experienced by pedestrians when going through those paths (weight-averaged by impedance and trip importance). Finally, it maps that performance. 


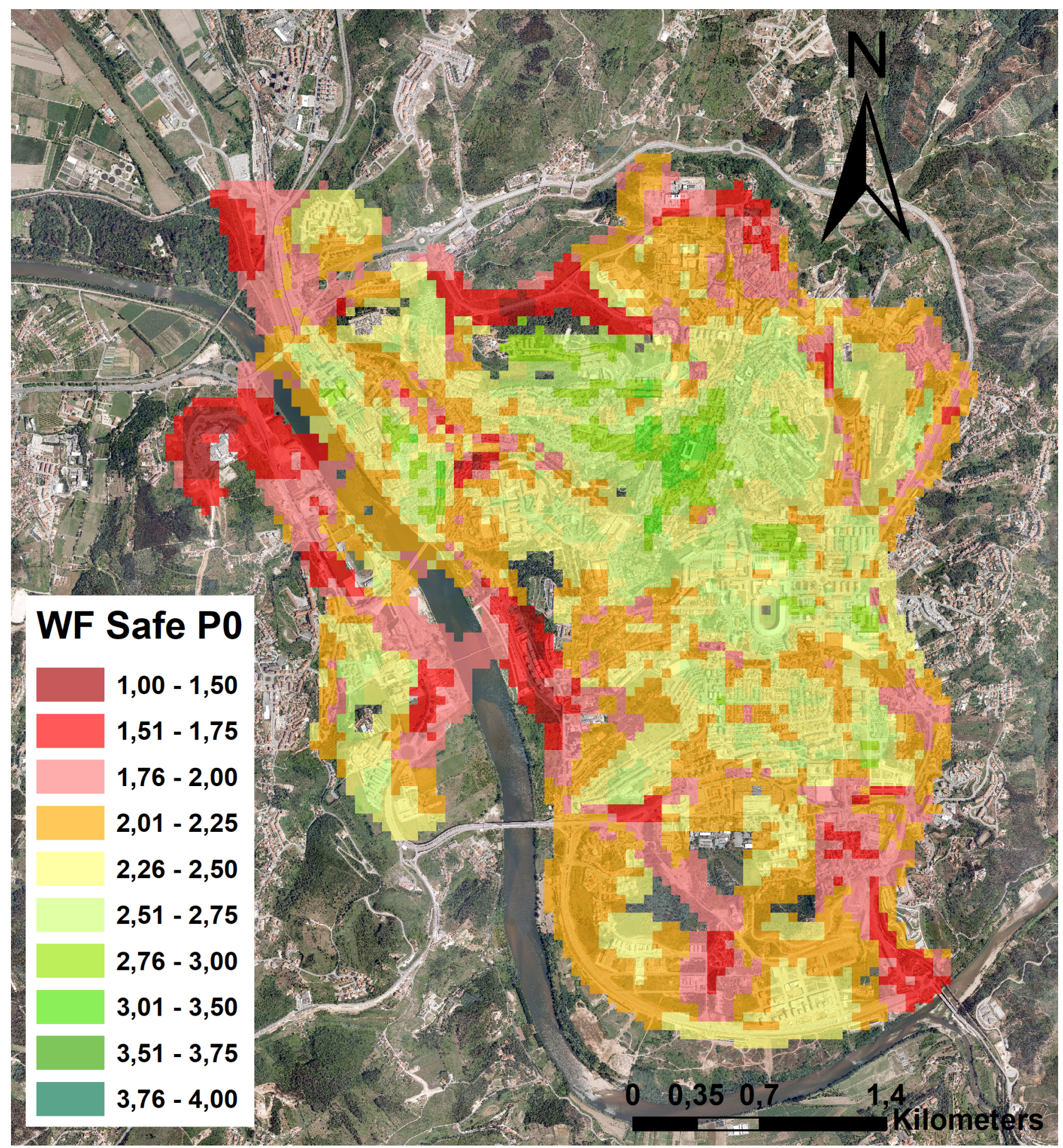

Figure 3. WF index with distance as impedance.

\subsection{Penalised distance as impedance}

Using distance as impedance means that pedestrians will choose the shortest route to destination, regardless of its performance, i.e. they will endure any degree of inconvenience coming from pedestrian infrastructure degradation to pursue the shortest route. Because pedestrians may instead decide to take small detours to avoid arcs with poor performance, a second type of impedance was considered, namely penalised distance. The findings of Ferrer et al. (2015) suggest that indeed factors such as e.g. sidewalk condition may influence pedestrian route choice. 
Penalised impedance was defined by multiplying, for every arc, its length by the penalty factor $[1+0.25$ $\times(4-P)]$ and adding the result to the pedestrian network feature class using GIS scripting tools. This factor is equivalent to increasing arc length up to $+75 \%$, depending on its performance.

Redoing calculations with penalised impedance (step 4) and reapplying the remaining steps of the methodology yielded results that are slightly better for this second impedance (expectable, since pedestrians thread better paths), but the main characteristics are essentially the same. Note that in practice pedestrians walk longer distances with this second impedance, but this does not show up in the WF index, as the index only maps network performance, not impedance or distance.

A statistical comparison between penalised and non-penalised distance WF values shows that, overall, differences are quite small. For $95 \%$ of the origins, the WF index does not improve more than 0.30 . The $5 \%$ origins with differences larger than 0.30 lie mostly in zones with long streets which call for performance improvements. No origin exhibits improvements greater than 1 performance class. Actually, only very few origins even come close to this value.

A map was also obtained but is very similar to that of Figure 3, so it is not shown. Either of them can help municipal decision makers planning for improvements. For this case study, results hint at the need to improve on the safety of some major arteries, providing for more separation between pedestrians and motor traffic. Since infrastructural improvements are known to effectively promote an increase in active traveling (see e.g. Panter et al., 2016; Longo et al., 2015), the WF index concept effectively helps identifying opportunities for such improvements, in a way which considers not just infrastructure condition but also, and perhaps more importantly, its impact on pedestrian accessibility.

\subsection{Other impedances}

Another possible impedance type is energy expenditure, i.e. muscular energy required to cross each arc. This impedance type can be useful for hilly cities and is, as mentioned, an example of a directed impedance. Time is also another natural (directed) impedance, for which one can consider more complex network analysis features, such as turns (additive costs to cumulative impedance at intersections). Carrying out WF calculations with these impedances would require endowing the pedestrian network with extra information such as e.g. energy, time for crossing each arc, etc.). For time as impedance, waiting times at intersections would also be needed (turn penalties).

\section{Conclusions and future work}

In this article, the concept of pedestrian or walking friendliness was proposed and a methodology to calculate it, based on GIS, was presented and applied to a case study. Walking friendliness is a quality of walking indicator which combines accessibility concepts with pedestrian infrastructure performance, and can also be seen as a quantitative walkability indicator. It is a measure of the practical impact that network performance has on pedestrian trips, which is hard to evaluate looking only at individual arc performance scores.

Calculating the WF index of a city (or zone thereof) requires GIS information and actual performance scores, so city/zone scale network performance field surveys are necessary. Although this could be 
considered as a drawback of the methodology, one can argue 'there are no free lunches': accurate indicators require precise data, so it is unavoidable to undergo some data collection effort. The reward of the methodology is that gathering network performance data allows decision makers to use that information to a much greater effect than simply mapping such data. Nevertheless, the case study shows it is possible to undertake data collection for a mid-sized city in four persons-month, a figure which may assist estimating the human resources necessary for surveying in future applications of the methodology. Because pedestrian networks always share a vast majority of arcs with a city's streets network, it is also important that their performance evaluation includes space sharing aspects.

The proposed methodology is flexible, in that it allows for using any impedance type and can be easily adapted for using importance weights based on a per-origin basis. This allows for a more precise analysis but requires data on trip demand per origin, which can be obtained e.g. from demand modelling estimates. Other improvements include making importance weights dependant on $i$-to- $j$ distance decay.

\subsection{Acknowledgements}

This work was partially supported by the Portuguese Foundation for Science and Technology under project grant UID/Multi/00308/2019.

\section{References}

Aghaabbasi M, Moeinaddini M, Shah MZ, Asadi-Shekari Z (2017). A new assessment model to evaluate the microscale sidewalk design factors at the neighbourhood level. Journal of Transport and Health, 5:97-112. https://doi.org/10.1016/j.jth.2016.08.012

Aghaabbasi M, Moeinaddini M, Shah MZ, Asadi-Shekari Z, Kermani MA (2018) Evaluating the capability of walkability audit tools for assessing sidewalks. Sustainable Cities and Society, 37:475484, https://doi.org/10.1016/j.scs.2017.12.001

Banister D, Hickman R (2013) Transport futures: thinking the unthinkable. Transport Policy, 29:283293. http://dx.doi.org/10.1016/j.tranpol.2012.07.005

Boarnet MG, Joh K, Siembab W, Fulton W, Nguyen MT (2011) Retrofitting the suburbs to increase walking: evidence from a land-use-travel study. Urban Studies, 48(1):129-159. https://doi.org/10.1177/0042098010364859

Chatterjee K, Clark B, Martin A, Davis A (2017) The Commuting and Wellbeing. Study: Understanding the Impact of Commuting on People's Lives. UWE Bristol, UK. https://travelbehaviour.files.wordpress.com/2017/10/caw-summaryreport-onlineedition.pdf

Corazza MV, Di Mascio P, Moretti L (2016) Managing sidewalk pavement maintenance: A case study to increase pedestrian safety. Journal of Traffic and Transportation Engineering, 3(3):203-214. https://doi.org/10.1016/j.jtte.2016.04.001

Donovan J (2015) Overcoming the tyranny of distance: making walking and cycling possible and preferable. Municipal Engineer, 168(2):115-124. https://doi.org/10.1680/muen.14.00014

Ewing R, Handy S (2009) Measuring the unmeasurable: Urban design qualities related to walkability. 
Journal of Urban Design, 14(1):65-84. https://doi.org/10.1080/13574800802451155

Ferrer S, Ruiz T, Mars L (2015) A qualitative study on the role of the built environment for short walking trips. Transportation Research Part F: Traffic Psychology and Behaviour, 33:141-160. https://doi.org/10.1016/j.trf.2015.07.014

Forsyth A, Krizek KJ (2010). Promoting walking and bicycling: assessing the evidence to assist planners. Built Environment, 36(4):429-446. https://doi.org/10.2148/benv.36.4.429

Gallin N (2001). Quantifying pedestrian friendliness - Guidelines for assessing pedestrian level of service. Road and Transport Research, 10(1):47-55. (No DOI)

Geurs KT, Ritsema van Eck J (2001) Accessibility measures: review and applications. Rijksinstituut voor Volksgezondheid en Milieu (National Institute of Public Health and Environment), RIVM report 408505 006. https://rivm.openrepository.com/rivm/bitstream/10029/259808/3/408505006.pdf

Hope-Dunbar J, Huxford R (2005) Briefing: Walking and Cycling: An Action Plan. Municipal Engineer, 158(2):83-85. https://doi.org/10.1680/muen.2005.158.2.83

Kang L, Fricker JD (2016) Sharing urban sidewalks with bicyclists? An exploratory analysis of pedestrian perceptions and attitudes. Transport Policy, 49:216-225. https://doi.org/10.1016/j.tranpol.2016.04.013

Kelly CE, Tight MR, Hodgson FC, Page MW (2011) A comparison of three methods for assessing the walkability of the pedestrian environment. Journal of Transport Geography, 19(6):1500-1508. https://doi.org/10.1016/j.jtrangeo.2010.08.001

Khisty CJ (1994) Evaluation of pedestrian facilities: Beyond the level-of-service concept. Transportation Research Record, 1438:45-50. http://onlinepubs.trb.org/Onlinepubs/trr/1994/1438/1438-006.pdf

Koh PP, Wong YD (2015) Walking and cycling as an urban transport option in Singapore. Municipal Engineer, 168(2):106-114. https://doi.org/10.1680/muen.13.00033

Landis B, Vattikuti V, Ottenberg R, McLeod D, Guttenplan M (2001) Modeling the roadside walking environment: Pedestrian level of service. Transportation Research Record, 1773:82-88. https://doi.org/10.3141/1773-10

Liang H, Chen D, Zhang Q (2017) Walking accessibility of urban parks in a compact megacity. Urban Design and Planning, 170(2):59-71. https://doi.org/10.1680/jurdp.16.00030

Longo A, Hutchinson WG, Hunter RF, Tully MA, Kee F (2015) Demand response to improved walking infrastructure: A study into the economics of walking and health behaviour change. Social Science and Medicine, 143:107-116, https://doi.org/10.1016/j.socscimed.2015.08.033

Lwin KK, Murayama Y (2011) Modelling of urban green space walkability: Eco-friendly walk score calculator. Computers, Environment and Urban Systems, 35(5):408-420. https://doi.org/10.1016/j.compenvurbsys.2011.05.002

Manaugh K, El-Geneidy AM (2011) Validating walkability indices: How do different households respond to the walkability of their neighborhood? Transportation Research Part D: Transport and Environment, 16(4):309-315. https://doi.org/10.1016/j.trd.2011.01.009

Manaugh K, El-Geneidy AM (2013) Does distance matter? Exploring the links among values, 
motivations, home location, and satisfaction in walking trips. Transportation Research part A: Policy and Practice, 50:198-208. https://doi.org/10.1016/j.tra.2013.01.044

Melia S, Shergold I (2018) Pedestrianisation and politics: a case study. Transport, 171(1): 30-41. https://doi.org/10.1680/jtran.16.00104

Moniruzzaman M, Paez A, Morency C (2014) Compliance potential mapping: a tool to assess potential contributions of walking towards physical activity guidelines. BMC Public Health, 14(1):article 511. https://doi.org/10.1186/1471-2458-14-511

Moody S, Melia S (2015) Shared space - research, policy and problems. Transport, 167(6):384-392. https://doi.org/10.1680/tran.12.00047

Ory DT, Mokhtarian PL, Redmond LS, Salomon I, Collantes GO, Choo S (2004) When is commuting desirable to the individual? Growth and Change, 35(3):334-359. https://doi.org/10.1111/j.14682257.2004.00252.x

Panter J, Heinen E, Mackett R, Ogilvie D (2016) Impact of New Transport Infrastructure on Walking, Cycling, and Physical Activity. American Journal of Preventive Medicine, 50(2):e45-e53. https://doi.org/10.1016/j.amepre.2015.09.021

Parkin J (2018) Designing for Cycle Traffic: International principles and practice. ICE Publishing, Thomas Telford Ltd, London, UK. ISBN: 978-0-7277-6349-5

Rassafi AA, Mohajeri F (2017) Analysis of walking patterns in a pedestrian environment using cellular automata. Municipal Engineer, in press. https://doi.org/10.1680/jmuen.16.00050

Reggiani A, Bucci P, Russo G (2011) Accessibility and impedance forms: empirical applications to the german commuting network. International Regional Science Review, 34:230-252. https://doi.org/10.1177/0160017610387296

Saghapour T, Moridpour S, Thompson RG (2018) Enhancing active transport demand Modelling by incorporating accessibility measures. Cities, 78:206-215. https://doi.org/10.1016/j.cities.2018.02.015

Sousa N, Coutinho-Rodrigues J, Natividade-Jesus E (2017). Sidewalk Infrastructure Assessment Using a Multicriteria Methodology for Maintenance Planning. Journal of Infrastructure Systems, 23(4), NN. https://doi.org/10.1061/(ASCE)IS.1943-555X.0000362

Tight M (2016) Sustainable urban transport - the role of walking and cycling. Engineering Sustainability, 169(3)87-91. https://doi.org/10.1680/jensu.15.00065

TRL - Transportation Research Laboratory (2003). Pedestrian environment review system. Crowthorne, UK.

van Wee B (2016) Accessible accessibility research challenges. Journal of Transport Geography, 51:916. http://dx.doi.org/10.1016/j.jtrangeo.2015.10.018

Woodcock J, Banister D, Edwards P, Prentice AM, Roberts I (2007). Energy and transport. Lancet, 370(9592):1078-1088. https://doi.org/10.1016/S0140-6736(07)61254-9

Yang L, Wang B, Zhou J, Wang X (2018) Walking accessibility and property prices. Transportation Research Part D: Transport and Environment, 62:551-562. https://doi.org/10.1016/j.trd.2018.04.001 